\title{
PLASMONIC LIGHT TRAPPING FOR THIN FILM A-SI:H SOLAR CELLS
}

\author{
VIVIAN E. FERRY ${ }^{1,2}$, MARC A. VERSCHUUREN ${ }^{3}$, HONGBO B. T. LI ${ }^{4}$, EWOLD VERHAGEN ${ }^{1}$, \\ ROBERT J. WALTERS ${ }^{1}$, RUUD E. I. SCHROPP ${ }^{4}$, HARRY A. ATWATER ${ }^{2}$, ALBERT POLMAN ${ }^{1}$ \\ ${ }^{1}$ FOM INSTITUTE AMOLF, AMSTERDAM, THE NETHERLANDS \\ ${ }^{2}$ CALIFORNIA INSTITUTE OF TECHNOLOGY, PASADENA, CA, USA \\ ${ }^{3}$ PHILIPS RESEARCH, EINDHOVEN, THE NETHERLANDS \\ ${ }^{4}$ UNIVERSITY OF UTRECHT, UTRECHT, THE NETHERLANDS
}

\begin{abstract}
Here we discuss the design, fabrication, and simulation of ultrathin film $n-i-p$ a-Si:H solar cells incorporating light trapping plasmonic back reflectors which exceed the performance of $n$-i-p cells on randomly textured Asahi substrates. The periodic patterns are made via an inexpensive and scalable nanoimprint method, and are structured directly into the metallic back contact. Compared to reference cells with randomly textured back contacts and flat back contacts, the patterned cells exhibit higher short-circuit current densities and improved overall efficiencies than either reference case. Angle-resolved photocurrent measurements confirm that the enhanced photocurrents are due to coupling to waveguide modes of the cell. Electromagnetic modeling is shown to agree well with measurements, and used to understand further details of the device.
\end{abstract}

\section{INTRODUCTION}

Light trapping is a critical parameter for improving the efficiency of thin and ultrathin film silicon solar cells. Naturally, as the thickness of the active region is reduced, light trapping methods are necessary to offset the reduced photocurrent, particularly in the red portion of the spectrum [1]. Reductions in the requisite thickness are a pathway to reduced costs for most solar cell materials, as well as a route to higher open circuit voltages due to decreased bulk recombination [2]. For ultrathin a-Si:H cells, there is an additional performance benefit in the improved stability under light illumination [3]. This light degradation issue, the Staebler-Wronski effect, has to date been a major limiting barrier to the long term adoption of a-Si:H solar cell technology, and so techniques to achieve high efficiencies in small quantities of material are highly relevant.

The conventional technique for light trapping in thin film Si cells is to use a randomly textured interface to scatter light, either through texturing at the transparent front interface or through the $\mathrm{Ag} / \mathrm{ZnO}$ back reflector. The incident sunlight is scattered into the semiconductor layer over a distribution of angles, thereby increasing the path length and the photocurrents. A number of fabrication methods for producing random textures exist, including hot sputtering of $\mathrm{Ag}$, anisotropic etching of $\mathrm{ZnO}$, and atmospheric pressure chemical vapor deposition of $\mathrm{SnO}_{2}: \mathrm{F}$ [4-6].

Plasmonic nanostructures have recently received attention as an alternative means to trap light inside solar cells [78]. Noble metal particles, such as $\mathrm{Ag}$ and $\mathrm{Au}$, are known to be strong scatterers of incident radiation, particularly near the surface plasmon resonance where the conduction band electrons oscillate coherently. The scattering cross section of a plasmonic particle can be as high as 10 times larger than the geometrical cross section, meaning that $10 \%$ surface coverage of these nanostructures would scatter $100 \%$ of the incident light. The scattering cross sections are spectrally dependent, but the spectral position can be tuned through modification of the shape, size, noble metal choice, and surrounding material.

Thin and ultrathin film solar cells can also support waveguide modes that propagate in the plane of the cell. As these modes propagate they can be absorbed partially in the active semiconductor, with some losses in the surrounding layers [9]. The fraction of a given mode that results in useful photocurrent varies across the spectrum, and depends on the semiconductor, metal, and spacer layers chosen. The number of supported modes also depends on both the thickness of each of the layers and on the wavelength. If incident sunlight can be efficiently coupled into these waveguide modes, then the direction of absorption is orthogonal to the direction of carrier collection, enabling the use of thinner semiconductor layers.

These modes are momentum-mismatched from incident light, and so a scattering object or grating is necessary to couple incident sunlight into each waveguide mode. The shape and dimensions of the scattering object can be tuned to couple to specific modes [10]. For solar applications, these structures should naturally be tuned to couple to modes with a high absorption fraction in the semiconductor. For photovoltaics that seek to use either enhanced scattering or waveguide modes to improve absorption, the major challenges are to design broadband nanostructures that operate at wavelengths across the solar spectrum, and to design nanostructures that are effective at all angles of incident sunlight. Although designs often focus on improving absorption near the band edge of the semiconductor, where absorption is 
otherwise weak, enhancements near the peak of the solar spectrum in a given thickness of material are also important.

To date, many plasmonic solar cell designs utilize metal nanostructures deposited on the front interface of the cell after the device is fully fabricated as scatterers to increase

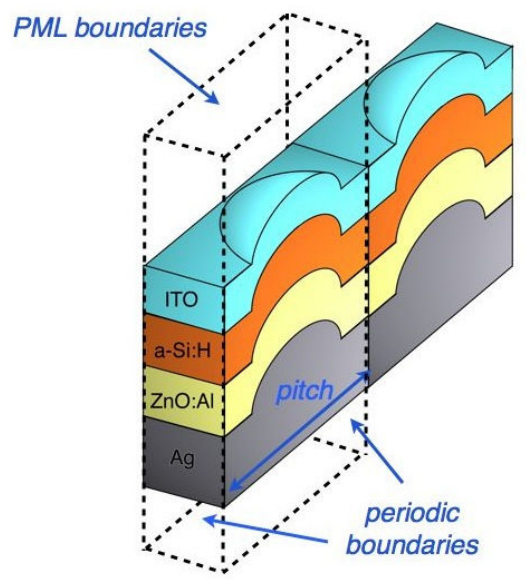

Figure 1 Schematic cross section of the a-Si:H solar cell design showing the unit cell and assumed boundary conditions for electromagnetic simulations.

absorption. Due to the high index of the semiconductor, the scatterers can be engineered to forward scatter sunlight into the semiconductor rather than backscatter the incident light into free space [11-13]. These designs have been shown to increase absorption by a factor of 18 near the band edge, but frequently suffer from losses in the blue side of the spectrum [14]. Other approaches embed the particles directly inside the semiconductor to enhance absorption through the high electric field intensities in the near field of the particle, but these particles must be carefully passivated to avoid additional losses due to their placement [15].

Our design avoids these losses by incorporating the nanostructures directly into the back contact of the cell $[16,17]$. The blue light is absorbed before reaching the metal scatterers, and the red light is trapped and scattered backwards into the absorber layer. Rather than an independent layer added to the top of a device, these patterns are used as the substrate, producing a conformal texture through all of the layers (Figs. 1 and 2). We have recently shown experimentally that a hole array on the back contact of an a-Si:H solar cell can increase the $J_{s c}$ by $26 \%$ compared to a flat reference cell. In this paper we describe arrays of nanopillars where we change both the pitch and the diameter, and demonstrate improved $\mathrm{J}_{\mathrm{sc}}$ 's over both flat and randomly textured reference cells.

\section{EXPERIMENTAL AND SIMULATION DETAILS}

Several different nanopatterns and reference cells were fabricated during the same deposition run to control for deposition variations. A master substrate was designed containing a variety of nanopatterns as well as flat reference cells, tiled with rotation to account for inhomogeneities. A second substrate of randomly textured Asahi U-type glass was included in the same deposition.

The nanopatterns were printed using a form of nanoimprint lithography, substrate conformal imprint lithography (SCIL) [18]. A master silicon wafer was first patterned using electron beam lithography, where the area of the total patterned area was $10 \mathrm{~cm}$ by $5 \mathrm{~cm}$, separated into $6 \mathrm{~mm}$ by $6 \mathrm{~mm}$ squares of distinct patterns. A composite PDMS stamp was molded from the master. The patterns were embossed into $100 \mathrm{~nm}$ of silica sol-gel on glass via the sequential evacuation of a system of grooves with 20 millibar pressure. The stamp was then removed via sequential re-pressurization. The sol-gel solidifies at room temperature while the stamp is in place, and is cured at $200{ }^{\circ} \mathrm{C}$ after stamp removal. The sol-gel is stable to at least $450{ }^{\circ} \mathrm{C}$, and non-absorbing. Both the master wafer and the stamp are reusable thousands of times.

The patterned silica was then sputter coated with $200 \mathrm{~nm}$ of $\mathrm{Ag}$ and $130 \mathrm{~nm}$ of $\mathrm{ZnO}: \mathrm{Al}$ to form a standard back contact. $160 \mathrm{~nm}$ of a-Si:H (total n-i-p thickness) was deposited by $13.56 \mathrm{MHz}$ plasma enhanced chemical vapor deposition on top of the patterned contacts, and $80 \mathrm{~nm}$ thick, $4 \mathrm{~mm} \times 4 \mathrm{~mm}$ pads of indium tin oxide (ITO) sputtered as a top contact. The nanopatterns were clearly visible through the ultrathin silicon, so the ITO pads were easily aligned to the bottom contacts. The thickness of the ITO was chosen to also act as an antireflective coating. A metal contact grid was evaporated over the ITO pads.

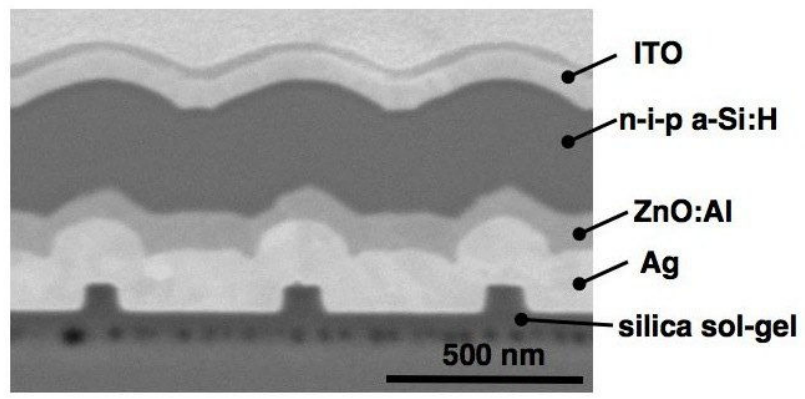

Figure 2 Cross section of cell with $\mathbf{3 4 0} \mathrm{nm}$ thick a-Si:H after fabrication showing that the layer depositions are highly conformal.

The topography of the resulting cells was investigated using atomic force microscopy (AFM) and focused ion beam (FIB) cross sections. Each deposition was conformal, so that the underlying pattern transferred through every layer, as illustrated in Figure 2. Both the top and back interfaces of the cell thus are therefore nanopatterned, with slightly different diameters due to the conformal coating. 
Measurements were taken under AM1.5 one sun illumination $\left(100 \mathrm{~mW} / \mathrm{cm}^{2}\right)$, and external quantum efficiency (EQE) measurements were taken using a Xenon lamp source through a monochromator with one sun light bias applied. EQE measurements were taken at both $V=0$ Volts and $V=-1$ Volts. Angle resolved EQE measurements were taken using a supercontinuum laser in combination with a monochromator.

\section{Pattern Design}

The patterns fabricated were square arrays of hemispherical particles, at two pitches (500 and $700 \mathrm{~nm}$ ) and four diameters $(200-275 \mathrm{~nm})$ as laid out in Figure 3 . The nanostructure is essentially a hemiellipsoidal particle after coating, and is $100 \mathrm{~nm}$ tall. Several copies of each of the 8 cells shown in Figure 3 were made on the master substrate, along with flat reference cells, to ensure that all measurements are a result of patterning and not of deposition inhomogeneity.

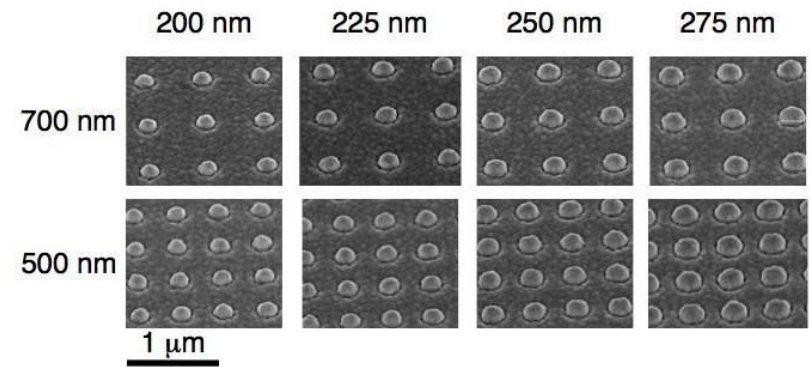

Figure 3 SEM images of nanopatterned Ag back contacts

\section{Electromagnetic Simulation}

Finite difference time domain (FDTD) simulations were performed using Lumerical software. The layer thicknesses were taken from the experimental cross sections, and the optical constants from previously measured $n, k$ data. The Ag was modeled using a LorentzDrude fit to Palik data [19]. For the patterned case, the nanostructures were assumed to be hemiellipsoids, and the unit cell was chosen to be the pitch between scatterers, with periodic boundary conditions as drawn in Figure 1. To model the randomly textured Asahi cell, AFM scans of the bottom interface were fed directly into simulation, and the successive layers were assumed to be completely conformal with identical texture. Periodic boundary conditions were used here as well, but the unit cell was chosen to be sufficiently large to minimize any coherent effects from the periodicity.

The $n$ and $p$ layers of the a-Si:H were neglected in the optical model, so the entire a-Si:H region is assumed to be intrinsic and optically active. The optical generation rate in the structure was calculated directly from the magnitude of the electric field $\mathbf{E}$ and the imaginary part of the permittivity for each material, $\varepsilon "$.

$$
G_{o p t}=\frac{1}{2 \mathrm{~h}} \varepsilon^{\prime \prime}|\mathbf{E}|^{2}
$$

The generation rate in each material can be isolated by mapping the spatially-dependent generation rate against the refractive index. Under the assumption that all photogenerated carriers are collected, this can be converted to a photocurrent, and the calculation at each wavelength can be weighted by the solar spectrum to determine a maximum achievable $\mathrm{J}_{\mathrm{sc}}$.

\section{RESULTS}

Table 1 shows a summary of the averaged electrical measurements for the $160 \mathrm{~nm}$ cells. As expected for devices with varying degrees of light trapping, the main difference between the devices is in the $\mathrm{J}_{\text {sc. }}$. Little difference is observed in JV measurements between the diameters, but there is strong dependence on pitch of the nanostructures. All of the patterned cells show a significant enhancement over the flat reference cell, increasing $\mathrm{J}_{\mathrm{sc}}$ by $30-50 \%$. Notably, the $\mathrm{J}_{\mathrm{sc}}$ of the $500 \mathrm{~nm}$ pitch cells exceeds that of the randomly textured Asahi cells, while the $700 \mathrm{~nm}$ pitch cells do not. The highest $J_{\mathrm{sc}}$ measured, which was in a cell with $275 \mathrm{~nm}$ diameter $\mathrm{Ag}$ with a $500 \mathrm{~nm}$ pitch, has a $\mathrm{J}_{\mathrm{sc}}$ of $11.8 \mathrm{~mA} / \mathrm{cm}^{2}$, vs. 10.8 $\mathrm{mA} / \mathrm{cm}^{2}$ for the best Asahi cell, a $J_{\mathrm{sc}}$ enhancement of $9.3 \%$.

The open circuit voltages and fill factors are similar across the substrate, indicating that a difference in semiconductor quality is not the origin of the difference between the Asahi cells and the nanopatterned cells. We do note that although the $\mathrm{J}_{\mathrm{sc}}$ decreases in the $160 \mathrm{~nm}$ thick cells, the efficiency of the best performing device in the $160 \mathrm{~nm}$ case and the $340 \mathrm{~nm}$ thick case are identical, and have an efficiency of $6.6 \%$. This is due to the increased $V_{\text {oc }}$ from decreased bulk recombination.

\begin{tabular}{|c|c|c|c|c|c|}
\hline & thickness & $\begin{array}{c}\mathrm{V}_{\text {oc }} \\
(\mathrm{V})\end{array}$ & $\begin{array}{c}\mathrm{J}_{\mathrm{sc}} \\
(\mathrm{mA} / \\
\left.\mathrm{cm}^{2}\right)\end{array}$ & $\mathrm{FF}$ & $\begin{array}{c}\text { Eff. } \\
(\%)\end{array}$ \\
\hline flat & 160 & 0.89 & 7.9 & 0.68 & 4.8 \\
\hline Asahi & 160 & 0.87 & 10.8 & 0.64 & 6.0 \\
\hline $500 \mathrm{~nm}$ pitch & 160 & 0.89 & 11.5 & 0.66 & 6.6 \\
\hline $700 \mathrm{~nm}$ pitch & 160 & 0.88 & 10.4 & 0.65 & 5.6 \\
\hline flat & 340 & 0.84 & 10.5 & 0.58 & 5.1 \\
\hline $500 \mathrm{~nm}$ pitch & 340 & 0.85 & 13.4 & 0.56 & 6.4 \\
\hline $700 \mathrm{~nm}$ pitch & 340 & 0.84 & 13 & 0.56 & 6.1 \\
\hline
\end{tabular}

Table 1 Summary of Electrical Measurements for measured cells.

\section{External Quantum Efficiency Measurements}

Figure 4 shows EQE measurements for several cells from the $160 \mathrm{~nm}$ thick a-Si:H deposition. The shape of the curves for the nanopatterned and reference cells are highly reproducible across the substrate. On the blue side 
of the spectrum, from $350-550 \mathrm{~nm}$, all four cases are nearly identical. The main difference in the photocurrent here is from $550-650 \mathrm{~nm}$, where the photocurrent from the $500 \mathrm{~nm}$ nanopatterned cell clearly exceeds the photocurrent from the randomly textured Asahi cell. From $650 \mathrm{~nm}-800 \mathrm{~nm}$ all of the nanopatterned and Asahi cells again exhibit comparable photocurrents, although they are all significantly higher than the photocurrent from the flat cell.

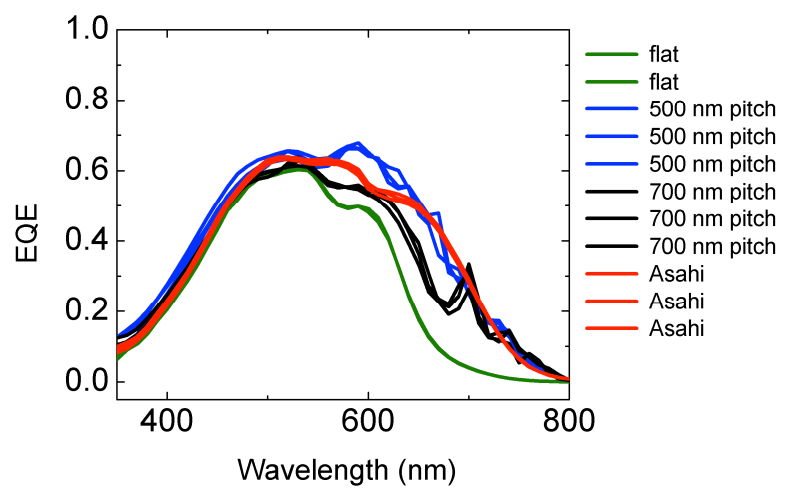

Figure 4 EQE measurements taken on multiple flat, randomly textured Asahi, and nanoimprinted cells across the substrate.

Comparing the shapes of the red side of the EQE curve gives further information about the observed photocurrent increases. Both the flat reference cell and the Asahi reference cell are extremely smooth across the spectral range where a-Si:H is absorbing. The nanopatterned cells exhibit several peaks, which sharpen at longer wavelengths. These peaks are reproducible for all cells of each pitch, so we attribute them to periodic waveguide modes of the structure. When the a-Si:H is strongly absorbing, these features will be broadened, and as the absorption decreases at longer wavelengths the modes will appear in more discrete bands of enhanced photocurrent.

To investigate the nature of these modes, we measured angle-resolved external quantum efficiencies for the Asahi and nanopatterned cells, shown in Figure 5. The Asahi cell exhibits an extremely isotropic response with changing angle. Fig. 5(b) shows the $500 \mathrm{~nm}$ pitch cell, which shows several interesting features which sharpen at longer wavelength. The background of Fig. 5(c) shows the photocurrent enhancement, or Fig. 5(b) / 5(a). From 550 650 there is a broad and essentially dispersionless enhancement over the randomly textured cell. From 650 $850 \mathrm{~nm}$ the features become sharper and there is stronger enhancement, although only at discrete wavelengths. Overlaid on Fig. 5(c) is a calculation of the modes supported by this structure and their position vs. angle. The calculations explain many of the crossings and broad features observed, particularly in the $650-750 \mathrm{~nm}$ range, indicating that the enhanced photocurrent is due to coupling to waveguide modes. From $550-650 \mathrm{~nm}$ there are many modes supported, and the absorption is very high, leading to broadening. Additionally, there are broad some features that are not well explained by the mode calculation; these may be due to localized enhancements in the vicinity of the scatterer. Weighting each angle by the solar spectrum shows that the nanopatterned cell exceeds the randomly textured cell up to $25^{\circ}$.
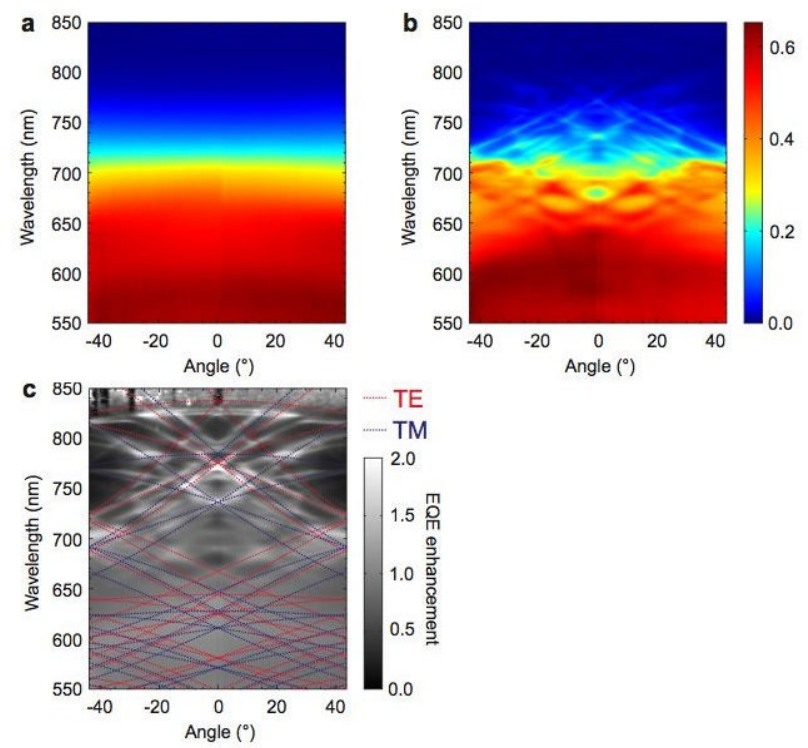

Fig. 5 Measured angle-resolved EQE for $160 \mathrm{~nm}$ thick a-Si:H cells. (a) Asahi cell (b) $500 \mathrm{~nm}$ pitch cell (c) photocurrent enhancement (Panel b/Panel a) with calculated overlaid modes.

\section{Electromagnetic Simulation}

Figure 6 shows a comparison between the measured EQE of a $500 \mathrm{~nm}$ pitch cell with a $300 \mathrm{~nm}$ diameter Ag scatterer and the calculated generation rate for a simulated structure using FDTD. The calculation was done as described above, assuming that the scatterers are perfect hemispheres. The layer thicknesses and degree of broadening for each deposited layer were approximated from the experimental cross sections. Figure 7 shows simulation vs. experimental data for the cells made on the Asahi substrate. As mentioned above, the Asahi cell was simulated using measured AFM data of the Ag-coated Asahi, and for simplicity we assumed no broadening as additional layers are deposited. In both cases many of the essential features are well reproduced by this curve, with peaks in the same locations if not exactly the same in magnitude. The differences between the model and experiment are likely to be due to differences in the assumed geometry and the measured vs. actual optical constants.

Electromagnetic simulation allows us to plot other interesting data, such as cross sectional maps of the generation rate at specific wavelengths. Figure 8 shows generation rate maps for the $500 \mathrm{~nm}$ pitch nanopatterned cell and the Asahi cell at two individual simulation 
wavelengths, $500 \mathrm{~nm}$ and $670 \mathrm{~nm}$. The simulations are fully three-dimensional, but only two-dimensional cross sections are shown in these images. To calculate the curves shown in Figures 6 and 7, the generation rate is integrated over only the portion containing a-Si:H, but here we show absorption in all of the layers, as it is instructive to identify the loss mechanisms.

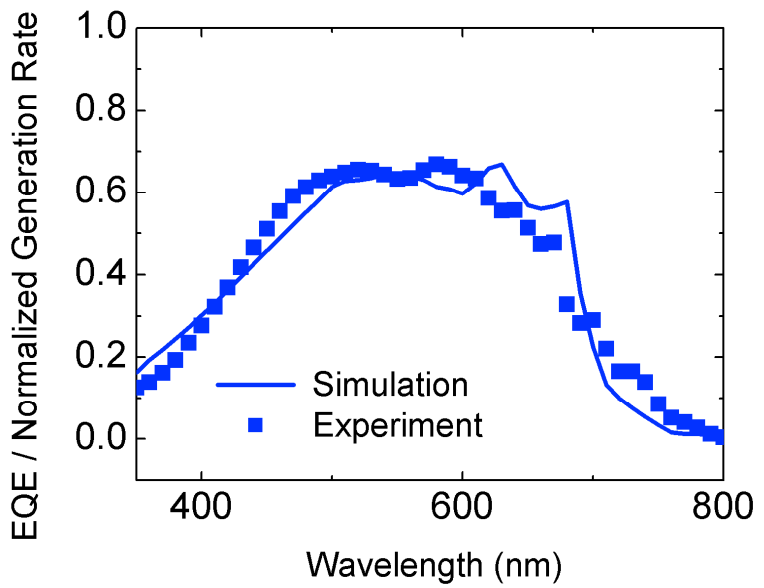

Figure 6 Comparison of measured EQE (squares) and simulated generation rate (line) for cells with $160 \mathrm{~nm}$ thick a-Si:H and $300 \mathrm{~nm}$ diameter Ag scatterers.

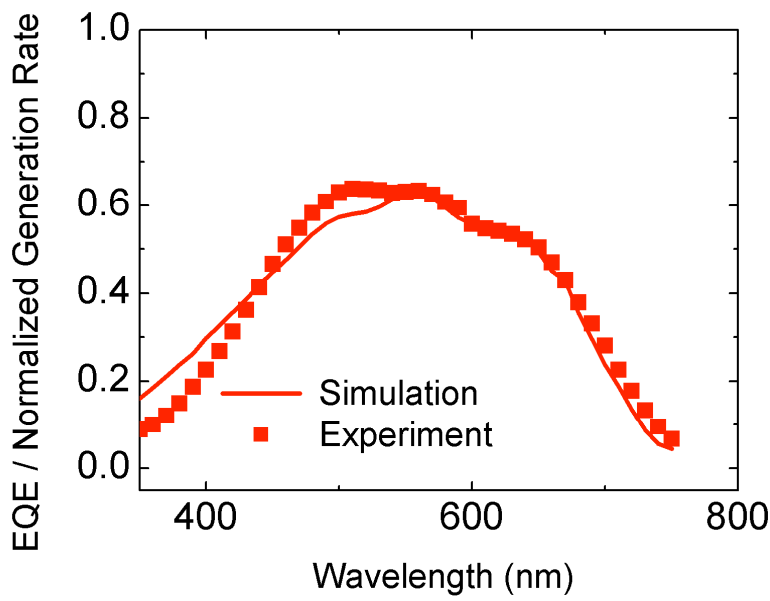

Figure 7 Comparison of measured EQE (squares) and simulated generation rate (line) for cells with $160 \mathrm{~nm}$ thick a-Si:H with Asahi texture.

In all four images, it is clear that the $\mathrm{ZnO}: \mathrm{Al}$ layer is very low loss and that the a-Si:H is the most strongly absorbing layer. The ITO and the Ag both have significant parasitic absorption, the latter particularly at $\lambda=670 \mathrm{~nm}$. It is also clear that the periodic structures show much more regular features of light absorption than the Asahi textured cell. For the nanopatterned cell, the main difference between the two wavelengths is the clear modal shape observed in the longer wavelength; at $\lambda=500 \mathrm{~nm}$ the absorption is almost entirely in the top portion of the cell. In the randomly textured cells, both the absorption in the a-Si:H and the parasitic absorption in the $\mathrm{Ag}$ tends to be concentrated around the tips of the convex portions.
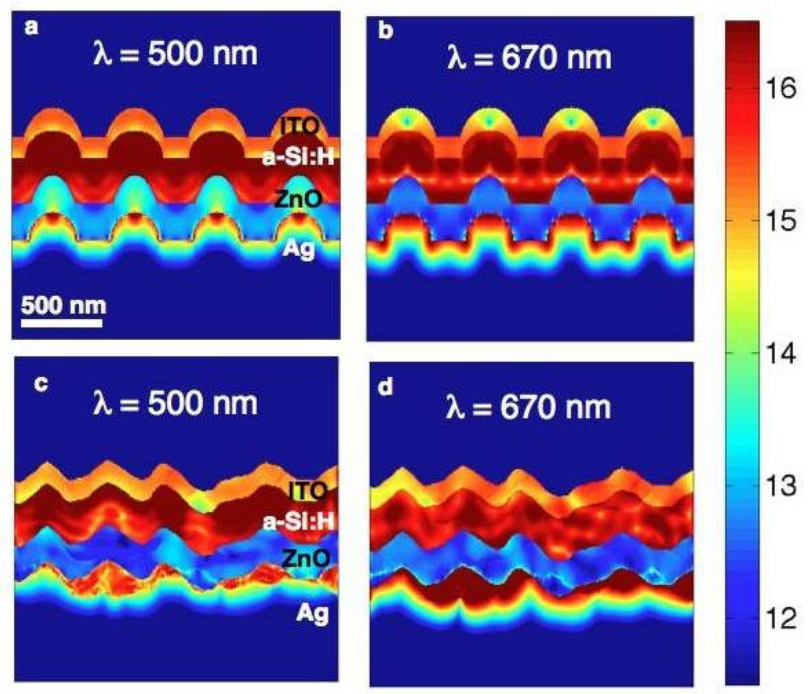

Figure 8 Generation rate maps of the simulated nanopatterned ( $a$ and $b$ ) and Asahi cells (c and d) at two different wavelengths. The color scale is logarithmic in electrons $\mathrm{sec}^{-1} \mathrm{~cm}^{-3}$.

\section{SUMMARY}

In summary, we have demonstrated plasmonic nanopatterned ultrathin film solar cells with photocurrents and efficiencies exceeding that of randomly textured cells. Through analysis of the EQE and angle-resolved EQE curves, we are able to identify that the photocurrent enhancement is due largely to coupling to waveguide modes. Electromagnetic simulation agrees well with the experimental measurements, and we are able to use it as a tool to visualize both current-generating and parasitic current. These methods should guide research toward the design of optimized light trapping nanostructures.

\section{ACKNOWLEDGEMENT}

We are grateful to C. H. M. van der Werf for solar cell deposition, and to L. A. Sweatlock, I. M. Pryce, and R. de Waele for illuminating discussions. The Caltech portion of this work was supported by the Department of Energy under contract number DE-FG02-07ER46405 (modeling) and DE-FG36-08G018006 (fabrication). Work at AMOLF is part of the research program of FOM which is financially supported by NOW. The work at Utrecht University was fully supported internally. This work is part of the SmartMix program Memphis and the Global Climate and Energy Project (GCEP). 


\section{REFERENCES}

[1] R. E. I . Schropp, M. Zeman. Amorphous and microcrystalline silicon solar cells: modeling, materials, and device technology, Kluwer Academic Publishers, Norwell Mass. 1998.

[2] P. Campbell, M. A. Green. "The limiting efficiency of silicon solar cells under concentrated sunlight", IEEE Trans. Electron. Dev. 33, 1986, pp. 234-239.

[3] D. L. Staebler, C. R. Wronski. "Reversible conductivity changes in discharge-produced amorphous silicon", Appl. Phys. Lett. 31, 1977, pp. 292-294.

[4] R. H. Franken, et al. "Understanding light trapping by light scattering textured back electrodes in thin film n-i-p type silicon solar cells", J. Appl. Phys. 102, 2007, 014503.

[5] U. Dagkaldiran, et al. "Amorphous silicon solar cells made with SnO2:F TCO films deposited by atmospheric pressure CVD", Mater. Sci. Eng. B, 159-160, 2009, pp. 69.

[6] J. Krč, F. Smole, M. Topič. "Potential of light trapping microcrystalline silicon solar cells with textured substrates", Prog. Photovolt. Res. Appl. 11, 2003, pp. 429436.

[7] H. A. Atwater and A. Polman. "Plasmonics for Improved photovoltaic devices”, Nat. Mater. 9, 2010, pp. 205-213.

[8] K. R. Catchpole and A. Polman. "Plasmonic solar cells", Opt. Exp. 16, 2008, pp. 21793-21800.

[9] P. N. Saeta, et al. "How much can guided modes enhance absorption in thin solar cells?" Opt. Exp. 17, 2009, pp. 20975-20990.

[10] V. E. Ferry, et al. "Plasmonic nanostructure design for efficient light coupling into solar cells", Nano Lett. 8, 2008, pp.4391-4397.

[11] K. R. Catchpole, A. Polman. "Design principles for particle plasmon enhanced solar cells", Appl. Phys. Lett. 93, 2008, 191113.

[12] H. R. Stuart, D. G. Hall. "Island size effects in nanoparticle-enhanced photodetectors", Appl. Phys. Lett. 73, 1998, pp. 3815-3817.

[13] D. Derkacs, et al. "Improved performance of amorphous silicon solar cells via scattering from surface Plasmon polaritons in nearby metallic nanoparticles", Appl. Phys. Lett. 89, 2006, 093103.

[14] F. J. Beck, A. Polman, K. R. Catchpole. "Tunable light trapping for solar cells using localized surface plasmons", J. Appl. Phys. 105, 2009, 114310.
[15] B. P. Rand, P. Peumans, S. Forrest. "Long-range absorption enhancement in organic tandem thin-film solar cells containing silver nanoclusters", J. Appl. Phys. 96, 2004, pp. 7519-7526.

[16] V. E. Ferry, et al. "Improved red-response in thin film a-Si:H solar cells with soft-imprinted plasmonic back reflectors", Appl. Phys. Lett., 95, 2009, 183503.

[17] V. E. Ferry, et al. "Light trapping in ultrathin plasmonic solar cells”, Opt. Exp. 18, 2010, pp. A237-A245.

[18] M. Verschuuren, $H$. van Sprang. "3D photonic structures by sol-gel imprint lithography”, Mater. Res. Soc. Sym. Proc. 1002, 2007, pp. N03-N05.

[19] A. D. Rakic, et al. "Optical properties of metallic films for vertical-cavity optoelectronic devices", Appl. Opt. 37, 1998, pp. 5271-5283. 